\title{
The anharmonic electron-phonon problem
}

\author{
J. K. Freericks ${ }^{1}$, Mark Jarrell ${ }^{2}$, and G. D. Mahan ${ }^{3}$ \\ ${ }^{1}$ Department of Physics, Georgetown University, Washington, DC 20057-0995 \\ ${ }^{2}$ Department of Physics, University of Cincinnati, Cincinnati, OH 45221 \\ ${ }^{3}$ Department of Physics and Astronomy, University of Tennessee, Knoxville, Tennessee 37996-1200 and Solid State Division, \\ Oak Ridge National Laboratory, P. O. Box 2008, Oak Ridge, Tennessee 37831-6030
}

(June 24, 2021)

The anharmonic electron-phonon problem is solved in the infinite-dimensional limit using quantum Monte Carlo simulation. Charge-density-wave order is seen to remain at half filling even though the anharmonicity removes the particle-hole symmetry (and hence the nesting instability) of the model. Superconductivity is strongly favored away from half filling (relative to the charge-density-wave order) but the anharmonicity does not enhance transition temperatures over the maximal values found in the harmonic limit.

Introduction Lattice anharmonicity is responsible for many different properties of solids. For example, the expansion of a crystal upon heating is due entirely to anharmonic effects - harmonic crystals do not change their volume upon heating. In addition, the phonon contribution to thermal resistivity is due, in some measure, to anharmonic effects. Furthermore, lattice modes in a real crystal are never completely harmonic. Anharmonic terms always enter to higher-order when expanding in the amplitude of the lattice vibrations.

But the effects of anharmonicity on the electronphonon problem, and more specifically on superconductivity, are relatively unknown. This is surprising, because the electron-phonon problem is well studied, especially in the weak coupling limit. Here the theory of electrons interacting with harmonic phonons, initially pioneered by Migdal [1] and Eliashberg [2], has led to an accurate description of superconductivity in most low-temperature phonon-mediated superconductors. It has been found, that in the regime where the electron-phonon coupling is weak so that the Migdal-Eliashberg theory still applies to the anharmonic case, the superconducting transition temperature is not enhanced by the presence of lattice anharmonicity 3. The situation as the coupling is increased is less clear. Recently, a new mechanism for superconductivity, driven by kinetic-energy effects, has been hypothesized in anharmonic crystals that display stronger electron-phonon coupling [4]. In this new mechanism, paired holes, in a nearly filled band, are more

\footnotetext{
(C) 1996 by the authors. Reproduction of this article by any means is permitted for non-commercial purposes.
}

mobile than single holes, driving the superconductivity.

An exact treatment of lattice anharmonicity is, however, difficult from a theoretical point of view because an anharmonic "perturbation" is never small - the phonon wavefunctions are always dominated by the anharmonic terms in the potential as the phonon coordinate becomes large. All that is known rigorously about the anharmonic electron-phonon problem is that the ground state must contain a spin singlet [5] for even numbers of electrons on a finite lattice.

Much progress can be made in the limit of large spatial dimension [6] where the lattice many-body problem can be mapped onto a self-consistently embedded impurity problem that is solved via a quantum Monte Carlo (QMC) simulation.

Model The simplest electron-phonon model that includes anharmonic effects is the anharmonic Holstein model [7] in which the conduction electrons interact with local phonon modes:

$$
\begin{aligned}
H & =-\sum_{i, j, \sigma} t_{i j} c_{i \sigma}^{\dagger} c_{j \sigma}+\sum_{i}\left(g x_{i}-\mu\right)\left(n_{i \uparrow}+n_{i \downarrow}\right) \\
& +\frac{1}{2 M} \sum_{i} p_{i}^{2}+\frac{1}{2} M \Omega^{2} \sum_{i} x_{i}^{2}+\alpha_{a n h} \sum_{i} x_{i}^{4}
\end{aligned}
$$

where $c_{i \sigma}^{\dagger}\left(c_{i \sigma}\right)$ creates (destroys) an electron at site $i$ with spin $\sigma, n_{i \sigma}=c_{i \sigma}^{\dagger} c_{i \sigma}$ is the electron number operator, and $x_{i}\left(p_{i}\right)$ is the phonon coordinate (momentum) at site $i$. The hopping of electrons is restricted to nearestneighbor lattice sites $i$ and $j$ and is governed by the hopping matrix element $t_{i j}\left(t_{i j}\right.$ is a Hermitian matrix).

The local phonon has a mass $M$ and a frequency $\Omega$ associated with it. The anharmonic contribution to the phonon potential energy is chosen to be a quartic in the phonon coordinate with a strength $\alpha_{a n h}$. The deformation potential (electron-phonon interaction strength) is parameterized by an energy per unit length and is denoted $g$. A useful combination of fundamental parameters is the bipolaron binding energy (in the harmonic limit) $U:=-g^{2} / M \Omega^{2}$ which determines the energy scale for the effective electron-electron interaction mediated by the phonons. The chemical potential is $\mu$.

The hopping $t_{i j}$ is isotropic with strength $t=: t^{*} / 2 \sqrt{d}$ where $t^{*}=1$ to define the energy scale and $d$ is the dimensionality. The mass is then set equal to one $(M=$ 1) leaving $U, \alpha_{a n h}, \Omega$, and the electron filling as free 
parameters. We set $\Omega=0.5 t^{*}$ which is approximately an order of magnitude smaller than the effective bandwidth $\left(W \approx 4 t^{*}\right)$, and we set $U=-4 t^{*}(g=1)$ which is well into the strongly coupled regime in the harmonic limit.

Results The original Holstein Hamiltonian corresponds to the case $\alpha_{a n h}=0$. Both the harmonic Holstein model and the harmonic Holstein-Hubbard model have been solved exactly in the limit of infinite dimensions via quantum Monte Carlo simulation [8,9]. These models display charge-density-wave (CDW) order near half filling and superconductivity (SC) away from half-filling. As the phonon frequency is increased, the $\mathrm{SC}$ is favored relative to the CDW order. However, in the strong-coupling limit, CDW order is favored over SC because of the bandnarrowing effect of the bipolaron. The quantum Monte Carlo simulations also found that the effective phonon potential (determined after integrating out the effects of the electrons) generically acquires a double-well structure signifying the high-temperature formation of a bipolaron.

An initial analysis of the anharmonic model can be made by employing the Born-Oppenheimer approxima-

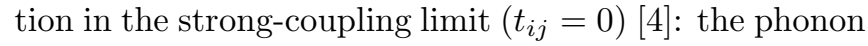
frequency is assumed to be smaller than any of the other energy scales so the phonons can be approximated by static lattice distortions corresponding to the minimum of the local phonon potential energy. Since the phonons couple linearly to the electronic charge, the equilibrium phonon coordinate varies when there are zero, one, or two electrons on a site. The origin $x_{0}=0$ is chosen to correspond to the case with no electrons on a site. Then $x_{1}$ and $x_{2}$ denote the equilibrium coordinates with one or two electrons on a lattice site. In the harmonic case the relative distances $x_{1}-x_{0}$ and $x_{2}-x_{1}$ are identical, which is a requirement for particle-hole symmetry. When a lattice anharmonicity is turned on, the equilibrium phonon coordinates with one and two electrons on a lattice site all move toward the origin, but the relative distances are no longer symmetric, rather the distance $x_{2}-x_{1}$ becomes significantly smaller than $x_{1}-x_{0}$, as can be seen in Figure 1(a).

The lattice anharmonicity and subsequent asymmetric shift of the equilibrium phonon coordinate have two main effects: (1) the model loses particle-hole symmetry which may allow a new type of kinetic-energy driven superconductivity to emerge [4] and (2) the effective electronelectron attraction is dramatically reduced as can be seen by a plot of the bipolaron binding energy in Figure 1(b). Thus the lattice anharmonicity generates an effective retarded repulsive interaction between the electrons. It is possible that this effective repulsive interaction between the electrons is sufficiently strong to destroy the kinetic-energy driven superconductivity. The lattice anharmonicity also breaks particle-hole symmetry, removing the nesting instability of the CDW at half filling and weak coupling. Hence, in the weak-coupling limit, one expects the lattice anharmonicity to favor $\mathrm{SC}$ relative to
CDW order, possibly completely suppressing CDW order, although it is also likely that anharmonic effects will reduce all transition temperatures. What is surprising is that a lattice anharmonicity as small as $\alpha_{a n h}=0.01$ (cf. Fig. 1(b)) can have such a large effect on the electronphonon problem in the strongly coupled regime studied here.

The anharmonic Holstein model is solved exactly in the infinite-dimensional limit via QMC simulation. The algorithm is straightforward, and has been described numerous times before 10,8,9, so it will not be repeated here. The QMC algorithm determines the single and two-particle Green functions at each of the Matsubara frequencies, which allows one to construct directly the momentum-dependent susceptibility to either CDW or $\mathrm{SC}$ order of the infinite-dimensional lattice. Divergence of either of these susceptibilities signals a transition to an ordered state.

We find that at half filling the system orders in a commensurate CDW phase. The results of the QMC simulation are presented in Figure 2. The solid dots represent the QMC transition temperatures, and the dashed line is a guide to the eye. The solid line is the result of a strong-coupling perturbation theory expansion 11 (fourth-order perturbation theory in $t^{*}$ ) that predicts that the system evolves from CDW order (solid line) to $\mathrm{SC}$ order (dotted line). We find no evidence for such an evolution in the QMC solution. Rather, the system remains in the CDW-ordered state even though there is no particle-hole symmetry remaining in the model. It is possible that as the strength of the anharmonicity is increased further that the SC order may overtake the CDW order, but it would be difficult to see that behavior with the QMC simulations because the transition temperatures become too small to be reliably determined. Note that the strong-coupling perturbation theory is accurate in predicting the peak position and peak height for the maximal CDW transition temperature. This is similar to what happened in the harmonic case as a function of the coupling strength.

The initial rise in $T_{c}$ occurs because of the reduction in the bipolaron binding energy $E_{b i p}$ as the strength of the anharmonicity increases. In the strong-coupling regime, the transition temperature is inversely proportional to $E_{b i p}\left(T_{c} \propto t^{* 2} / E_{b i p}\right)$. As the bipolaron unbinds, the system evolves from preformed-pair physics to BCS-type physics where pair formation and condensation both occur at $T_{c}$. This then causes the transition temperature to drop as $\alpha_{a n h}$ is increased further.

This physics can be dramatically illustrated by extracting the effective phonon potential from the QMC simulation [8]. The probability distribution for the local phonon coordinate $x$ is measured for each of the imaginary time slices. This probability distribution is then equated to an activated form for an effective potential $P(x)=\exp \left[-V_{\text {eff }}(x) / T\right]$. The effective phonon potential 
includes the effects of the interaction of the electrons with the phonons. In the harmonic limit, this effective potential was seen to evolve from a harmonic form $\left(x-x_{1}\right)^{2}$ to a symmetric double-well form as the coupling strength increased [8]. The double well signified bipolaron formation, since the number of electrons sitting at the lattice site was either zero or two for each of the minima (centered around $x_{0}$ and $x_{2}$ ). The results for the effective phonon potential in the anharmonic model at half filling and $T=0.125 t^{*}$ are presented in Figure 3. The effective potential is plotted versus a rescaled phonon coordinate for different values of $\alpha_{a n h}$. The zeroes of the potentials are shifted vertically to separate the different plots. Note that the addition of anharmonicity squeezes the phonon coordinate toward the origin, and makes the effective phonon potential asymmetric (corresponding to the loss of particle-hole symmetry). The barrier height decreases as $\alpha_{a n h}$ increases, as required by the reduction of the bipolaron binding energy. Note that the maximum CDW $T_{c}$ occurs when the barrier height is on the order of $T_{c}\left(\alpha_{a n h} \approx 0.0075\right)$.

In order to find $\mathrm{SC}$ solutions, we must dope the system off half filling. The phase diagram for three different values of $\alpha_{a n h}$ is shown in Figure 4. In Figure 4(a), a small value of $\alpha_{a n h}=0.005$ is chosen. This result is close to the harmonic result. Nevertheless, even at this small value of $\alpha_{a n h}$, the phase diagram is not symmetric with respect to reflection about half filling, signifying the particle-hole asymmetry. We compare to the strong-coupling perturbation theory, which predicts CDW order to survive until doped well off of half filling. This approximation is, by construction, particle-hole symmetric. Note that the case of less than half filling is approximated much better by the perturbative calculation, than the greater than halffilling case.

Figure 4(b), has $\alpha_{a n h}=0.02$ which lies at a point beyond where the CDW $T_{c}$ is maximal. The perturbative approximation is less accurate here, but displays the correct order-of-magnitude estimate for the phase diagram. We see how the anharmonicity favors the superconducting solutions, pushing the CDW-SC phase boundary toward half filling. SC is favored because the squeezing of the phonon coordinate toward the origin enhances the Frank-Condon overlap factors associated with electronic hopping and increases the electronic bandwidth.

Figure 4(c), takes the large value $\alpha_{a n h}=0.1$. The strong-coupling theory fails in this regime, and so it has not been included in the plot. We see once again how the increase in $\alpha_{\text {anh }}$ now suppresses the transition temperatures, and how it moves the CDW-SC boundary closer to half filling. There is no evidence for the new SC mechanism.

We found no evidence for incommensurate CDW order, either at half filling or away from half filling. The CDW susceptibility still had it's maximum at the $(\pi, \pi, \pi, \ldots)$ point when the SC susceptibility diverged at a higher $T_{c}$.
Anharmonicity does not favor incommensurate order in this regime of parameter space.

The effect of anharmonicity on the electron-phonon problem has some aspects that are similar to, and some aspects that are different from the effect of a direct Coulomb repulsion 9. In both cases the superconducting order is favored by the additional interaction relative to the charge-density-wave order, in the sense that the SC-CDW phase boundary moves toward half filling. However, the anharmonicity breaks the particlehole symmetry, while the Coulomb repulsion does not. Furthermore, the CDW transition temperature is moderately suppressed even for large values of the anharmonicity. Whereas, if the Coulomb repulsion is increased to large enough values, the CDW $T_{c}$ will plummet to zero.

Conclusion We have analyzed the effect of anharmonicity on the electron-phonon problem in the regime where the harmonic model has formed bipolarons. We find that the anharmonicity breaks particle-hole symmetry and produces phase diagrams that are not symmetric about half filling. The anharmonicity acts in some respects as a retarded "Coulomb repulsion", since it greatly favors SC solutions relative to CDW ones. However, in the infinite-dimensional limit, we did not find any enhancement to $T_{c}$ 's for the anharmonic model relative to the maximal values found in the harmonic case. Furthermore, we found no evidence for any kind of novel superconducting mechanism driven by kinetic-energy effects. We also studied the effective phonon potential which includes the effects of the interaction of the phonon with the electrons. We discovered that the anharmonicity tends to squeeze the phonon coordinates closer to the origin, and rapidly suppresses the bipolaron formation. We also verified that the strong-coupling perturbation theory accurately describes the anharmonic Holstein model in the regime where the system still has preformed pairs.

We would like to acknowledge useful conversations with W. Chung, J. Hirsch and J. Serene. J. K. F. acknowledges the Donors of The Petroleum Research Fund, administered by the American Chemical Society, for partial support of this research (ACS-PRF\# 29623-GB6) and an Oak Ridge Associated University Junior Faculty Enhancement Award for partial support of this research. M. J. acknowledges support of NSF grants DMR-9406678 and DMR-9357199. G. D. M. acknowledges support by the University of Tennessee, and by Oak Ridge National Laboratory, managed by Lockheed Martin Energy Research Corporation for the U.S. Department of Energy under contract number DE-AC05-96OR22464. This research was also supported in part by grant number DMR950010P from the Pittsburgh Supercomputing Center, sponsored by the National Science Foundation. 
[1] A. B. Migdal, Zh. Eksp. Teor. Fiz. 34, 1438 (1958) [Sov. Phys.-JETP 7, 999 (1958)].

[2] G. M. Eliashberg, Zh. Eksp. Teor. Fiz. 38, 966 (1960) [Sov. Phys.-JETP 11, 696 (1960)].

[3] J. C. K. Hui and P. B. Allen, J. Phys. F 4, L42 (1974); A. E. Kavakozov and E. G. Maksimov, Zh. Eksp. Teor. Fiz. 74, 681 (1978) [Sov. Phys. JETP 47, 358 (1978)]; G. D. Mahan and J. O. Sofo, Phys. Rev. B 47, 8050 (1993).

[4] J. E. Hirsch, Phys. Rev. B 47, 5351 (1993); F. Marsiglio and J. E. Hirsch, Phys. Rev. B 49, 1366 (1994).

[5] J. K. Freericks and E. H. Lieb, Phys. Rev. B 51, 2812 (1995).

[6] W. Metzner and D. Vollhardt, Phys. Rev. Lett. 62, 324 (1989).

[7] T. Holstein, Ann. Phys. 8, 325 (1959).

[8] J. K. Freericks, M. Jarrell, and D. J. Scalapino, Phys. Rev. B 48, 6302 (1993); J. K. Freericks, M. Jarrell, and D. J. Scalapino, Europhys. Lett. 25, 37 (1994); J. K. Freericks and M. Jarrell, in Computer Simulation Studies in Condensed Matter Physics VII, edited by D. P. Landau, K. K. Mon, and H.-B. Schüttler, (Springer-Verlag, 1995).

[9] J. K. Freericks and M. Jarrell, Phys. Rev. Lett. 75, 2570 (1995).

[10] M. Jarrell, Phys. Rev. Lett. 69, 168 (1992).

[11] J. K. Freericks, Phys. Rev. B 48, 3881 (1993); J. K. Freericks and G. D. Mahan, submitted to Phys. Rev. B.

FIG. 1. (a) Phonon coordinates at the minima of the anharmonic phonon potential with zero $\left(x_{0}\right)$, one $\left(x_{1}\right)$, and two $\left(x_{2}\right)$ electrons on a site as a function of $\alpha_{a n h}$ in the atomic limit $\left(t_{i j}=0\right)$. (b) Reduction of the bipolaron binding energy as a function of $\alpha_{a n h}$ in the atomic limit $\left(t_{i j}=0\right)$.

FIG. 2. Charge-density-wave transition temperature at half filling as a function of $\alpha_{a n h}$. The QMC results (dots-no SC transitions were found at half filling with the QMC) are compared to the strong-coupling perturbation theory for CDW order (solid line) and SC order (dotted line). The dashed line through the QMC points is a guide to the eye.

FIG. 3. Effective phonon potential for the anharmonic Holstein model at half filling and $T=0.125 t^{*}$. The labels on the curves are the values of $\alpha_{a n h}$. The potentials are shifted by adding a constant to them to separate the curves.

FIG. 4. Phase diagrams for the anharmonic Holstein model at three different values of $\alpha_{a n h}$ : (a) 0.005 ; (b) 0.02; and (c) 0.1 . The solid dots are CDW phases, the open triangles are SC phases. The solid lines are a strong-coupling approximation to the CDW $T_{c}$, and the dotted lines are a strong-coupling approximation to the $\mathrm{SC} T_{c}$.

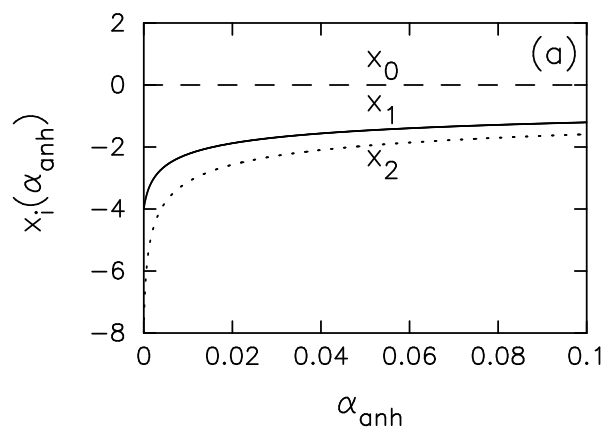

Freericks, Jarrell, and Mahan, Phys. Rev. Lett., Figure 1(a)

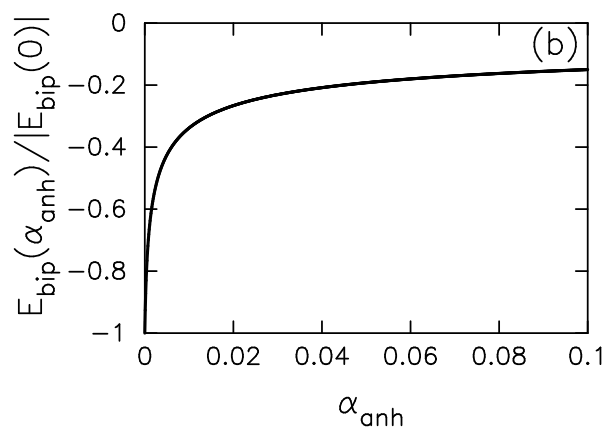

Freericks, Jarrell, and Mahan, Phys. Rev. Lett., Figure 1(b)

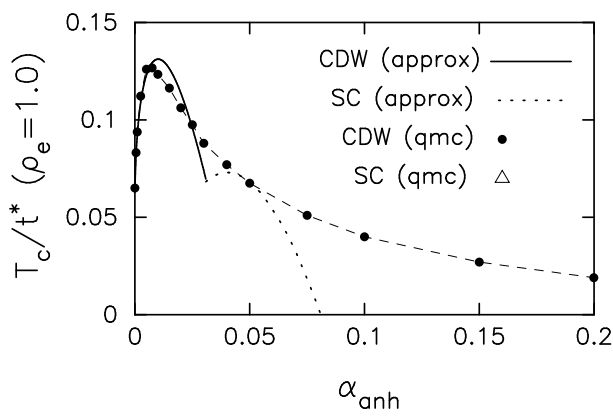

Freericks, Jarrell, and Mahan, Phys. Rev. Lett., Figure 2

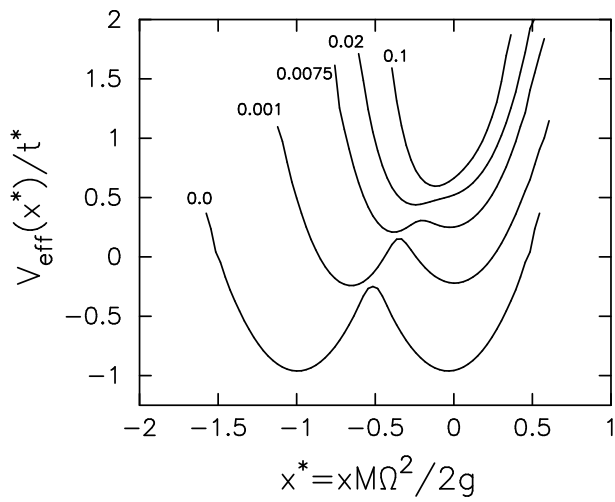

Freericks, Jarrell, and Mahan, Phys. Rev. Lett., Figure 3 

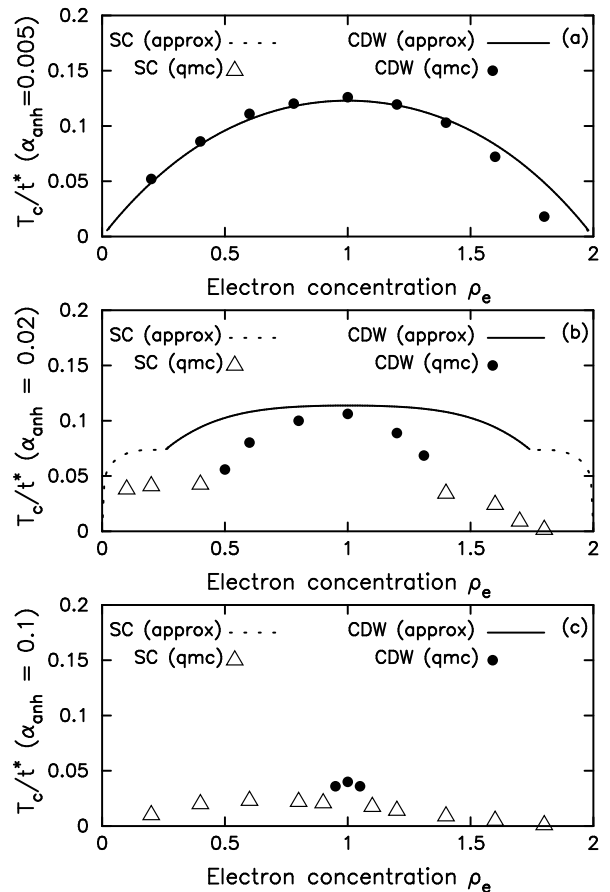

Freericks, Jarrell, and Mahan, Phys. Rev. Lett., Figure 4 